\title{
Leading coefficients of Kazhdan-Lusztig polynomials and fully commutative elements
}

\author{
R.M. Green
}

Received: 17 July 2008 / Accepted: 18 September 2008 / Published online: 9 October 2008

(C) Springer Science+Business Media, LLC 2008

\begin{abstract}
Let $W$ be a Coxeter group of type $\widetilde{A}_{n-1}$. We show that the leading coefficient, $\mu(x, w)$, of the Kazhdan-Lusztig polynomial $P_{x, w}$ is always equal to 0 or 1 if $x$ is fully commutative (and $w$ is arbitrary).
\end{abstract}

Keywords Kazhdan-Lusztig polynomials · Affine Weyl groups ·

Fully commutative elements $\cdot 0-1$ conjecture

\section{Introduction}

In their famous paper [4], Kazhdan and Lusztig showed how to associate to an arbitrary Coxeter group $W$ a remarkable family of polynomials in an indeterminate $q,\left\{P_{x, w}(q): x, w \in W\right\}$, which are now known as Kazhdan-Lusztig polynomials. The Kazhdan-Lusztig polynomials are of key importance in algebra and geometry. For example, they are intimately related to the geometry of Schubert varieties, and they are necessary for the statement of Lusztig's famous conjecture [8] regarding the characters of irreducible modules of reductive algebraic groups over a field of characteristic $p>0$.

The polynomial $P_{x, w}$ is zero unless $x \leq w$ in the Bruhat order on $W$. If we have $x<w$, then $P_{x, w}(q)$ is of degree at most $(\ell(w)-\ell(x)-1) / 2$, where $\ell$ is the length function on the Coxeter group. The cases where this degree bound is achieved are of particular importance, and in such cases, the leading coefficient of $P_{x, w}(q)$ is denoted by $\mu(x, w)$. The $P_{x, w}(q)$ and $\mu(x, w)$ are defined by recurrence relations and are very difficult to compute efficiently, even for some moderately small groups.

R.M. Green $(\bowtie)$

Department of Mathematics, University of Colorado, Campus Box 395, Boulder, CO 80309-0395,

USA

e-mail: rmg@euclid.colorado.edu 
As well as playing a key role in the computation of the polynomials $P_{x, w}(q)$, the $\mu$-function is also an interesting object in its own right. Empirical evidence had suggested that in the special case of the symmetric group (a Coxeter group of type $A$ ), the values $\mu(x, w)$ were always equal to 0 or 1 . This hypothesis, which was known as the 0-1 Conjecture, was proved to be false by McLarnan and Warrington [11]. However, as pointed out by B.C. Jones [3, Sect. 1], the values $\mu(x, w)$ in the symmetric group case are indeed equal to 0 or 1 if one restricts $x$ and/or $w$ to certain (interesting) classes of permutations. For example, we have $\mu(x, w) \in\{0,1\}$ if any of the following holds:

(a) the symmetric group $S_{n}$ in question satisfies $n \leq 9$ [11];

(b) $w$ is a covexillary permutation, meaning that the one-line notation for $w$ avoids the permutation pattern [3412] [6, 7];

(c) $a(x)<a(w)$ [15], where $a$ is Lusztig's $a$-function from [10];

(d) $w$ corresponds to a smooth Schubert variety [5].

(In case (d), the polynomials $P_{x, w}$ themselves are equal to 0 or 1.) The main result of [3] is that if $W$ is a finite Weyl group, then $\mu(x, w)$ is always 0 or 1 if $w$ is a Deodhar element; the latter are a subclass of the fully commutative elements.

In this paper, we make a restriction on $x$ rather than $w$, and we work in the larger Coxeter group $W=W\left(\widetilde{A}_{n-1}\right)$ of type $\widetilde{A}_{n-1}$. This group, which is infinite, naturally contains the symmetric group $S_{n}$ as a subgroup, and it can be thought of as a certain group of periodic permutations of the integers [9]. We will restrict $x$ to be a fully commutative element in the sense of Stembridge [14]. There are infinitely many such elements in the group $W$, and for each choice of $x$, there are infinitely many $w$ with $x \leq w$. Our main result is that if $x, w \in W$ and $x$ is fully commutative, then $\mu(x, w) \in$ $\{0,1\}$.

Computational evidence suggests that there may be a large class of Coxeter groups (possibly all of them) for which $\mu(x, w) \in\{0,1\}$ when $x$ is fully commutative. We intend to return to this question elsewhere.

\section{Definitions}

Let $n \geq 3$, and let $W=W\left(\widetilde{A}_{n-1}\right)$ be the Coxeter group of type $\widetilde{A}_{n-1}$ with the distinguished set of generating involutions

$$
S=\left\{s_{i}: 0 \leq i<n\right\}
$$

In other words, $W$ is given by the presentation

$$
\left.W=\langle S|\left(s_{i} s_{j}\right)^{m(i, j)}=1 \text { for } 0 \leq i, j<n\right\rangle,
$$

where we define $m(i, i)=1, m(i, j)=3$ if $|i-j|= \pm 1 \bmod n$, and $m(i, j)=2$ otherwise. It turns out [2, Proposition 5.3] that the elements of $S$ are distinct as group elements, and that $m(i, j)$ is the order of $s_{i} s_{j}$. The subgroup of $W$ generated by $S \backslash\left\{s_{0}\right\}$ is isomorphic to the symmetric group $S_{n}$; one isomorphism is given by the correspondence $s_{i} \leftrightarrow(i, i+1)$. 
Each element $w \in W$ can be written as a word $w=s_{i_{1}} s_{i_{2}} \cdots s_{i_{k}}$ with $s_{i j} \in S$. If this $k$ is minimal for a given $w$, then we call $k$ the length of $w$ and write $k=\ell(w)$. We call $s_{i_{1}} s_{i_{2}} \cdots s_{i_{k}}$ a reduced expression for $w$. More generally, we call a product $w_{1} w_{2} \cdots w_{k}$ of elements $w_{i} \in W$ reduced if $\ell\left(w_{1} w_{2} \cdots w_{k}\right)=\sum_{i} \ell\left(w_{i}\right)$. We write

$$
\mathcal{L}(w)=\{s \in S: \ell(s w)<\ell(w)\}
$$

and

$$
\mathcal{R}(w)=\{s \in S: \ell(w s)<\ell(w)\} .
$$

The set $\mathcal{L}(w)$ (respectively, $\mathcal{R}(w)$ ) is called the left (respectively, right) descent set of $w$. We call a (left or right) descent set commutative if it consists of pairwise commuting generators.

There is a partial order on $W$ called the (strong) Bruhat order and denoted by $\leq$. It is characterized by the property that if $t_{1} t_{2} \cdots t_{r}$ is a reduced expression for $w \in W$ with $t_{i} \in S$, then the set of elements $x \leq w$ are precisely those of the form $x=t_{i_{1}} t_{i_{2}} \cdots t_{i_{k}}$, where we have $1 \leq i_{1}<i_{2}<\cdots<i_{k} \leq r$.

For each $x, w \in W$, Kazhdan and Lusztig [4] defined a polynomial $P_{x, w} \in \mathbb{Z}[q]$. We have $P_{w, w}=1$, and unless $x \leq w$ in the Bruhat order, we have $P_{x, w}=0$. The other possibility is that $x<w$, in which case $P_{x, w}$ is a polynomial in $q$ of degree at most $(\ell(w)-\ell(x)-1) / 2$. We define $\mu(x, w)$ to be the (integer) coefficient of $q^{(\ell(w)-\ell(x)-1) / 2}$ in $P_{x, w}$; this can be nonzero only if (a) $x<w$ and (b) $\ell(x)$ and $\ell(w)$ have opposite parities. We write $x \prec w$ to mean that both $\mu(x, w) \neq 0$ and $x<w$. Using these facts, one can define the Kazhdan-Lusztig polynomials recursively via a recurrence formula proved in [4]. For this, we may assume that $w \neq 1$, for otherwise we have $x=1$ and $P_{x, w}=1$. We choose $s$ such that $\ell(s w)<\ell(w)$ (take $s$ to be the first letter in a reduced expression for $w$ ) and we define $v=s w$. We may then compute $P_{x, w}$ recursively by the formula

$$
P_{x, w}=q^{1-c} P_{s x, v}+q^{c} P_{x, v}-\sum_{\substack{z<v \\ s z<z}} \mu(z, v) q^{(\ell(w)-\ell(z)) / 2} P_{x, z},
$$

where we define $c=0$ if $x<s x$ and $c=1$ otherwise. Notice that a knowledge of the values of the $\mu$-function is important for computing the polynomials. However, as we shall see, it is sometimes possible to compute $\mu$ values without first computing the corresponding polynomials.

We call an element $w \in W$ complex if it can be written as a reduced product $x_{1} w_{i j} x_{2}$, where $x_{1}, x_{2} \in W,|i-j|= \pm 1 \bmod n$ and $w_{i j}=s_{i} s_{j} s_{i}=s_{j} s_{i} s_{j}$. Denote by $W_{c}$ the set of all elements of $W$ that are not complex. The elements of $W_{c}$ are the fully commutative elements of [14]; they are characterized by the property that any two reduced expressions may be obtained from each other by repeatedly transposing adjacent commuting generators.

A key concept for this paper is that of a star operation, which was introduced in [4, Sect. 4.1]. Let $I=\{s, t\} \subseteq S$ be a pair of noncommuting generators of $W$. Let $W^{I}$ denote the set of all $w \in W$ satisfying $\mathcal{L}(w) \cap I=\emptyset$. Standard properties of Coxeter groups [2, Sect. 5.12], show that any element $w \in W$ may be uniquely written as $w=w_{I} w^{I}$ reduced, where $w_{I} \in W_{I}=\langle s, t\rangle$ (the subgroup generated by $s$ and $t$ ) and $w^{I} \in W^{I}$. There are four distinct possibilities for elements $w \in W$ : 
(i) $w$ is the shortest element in the coset $W_{I} w$, so $w_{I}=1$ and $w \in W^{I}$;

(ii) $w$ is the longest element in the coset $W_{I} w$, so $w_{I}$ is the longest element of $W_{I}$ (which in this case implies that $W_{I} \cong S_{3}$ and $w_{I}=s t s=t s t$ );

(iii) $w$ is one of the two elements $s w^{I}$ or $t s w^{I}$;

(iv) $w$ is one of the two elements $t w^{I}$ or $s t w^{I}$.

The sets $\left\{s w^{I}, t s w^{I}\right\}$ and $\left\{t w^{I}, s t w^{I}\right\}$ appearing in (iii) and (iv) are called (left) $\{s, t\}$-strings, or strings if the context is clear. If $w$ is an element of an $\{s, t\}$-string, $S_{w}$, then we define ${ }^{*} w$ to be the other element of $S_{w}$. If $w$ is not an element of an $\{s, t\}$-string (in other words, case (i) or (ii) applies) then ${ }^{*} w$ is undefined.

There are also obvious right handed analogues to the above concepts, so the symbol $w^{*}$ may be used with the analogous meaning (with respect to a pair $I$ of noncommuting generators).

\section{Preparatory results}

In Sect. 3, we collect some results from the literature for use in the proof of our main result.

The following key properties of the $\mu$-function were proved in [4].

\section{Proposition 3.1}

(i) If $\ell(x)=\ell(w) \bmod 2$ or $x \not \leq w$, then we have $\mu(x, w)=0$.

(ii) Let $x, w \in W$ be elements of left $\{s, t\}$-strings (for the same $s$ and $t$, but possibly different strings). Then we have $\mu(x, w)=\mu\left({ }^{*} x,{ }^{*} w\right)$.

(iii) Let $x, w \in W$ be elements of right $\{s, t\}$-strings (for the same $s$ and $t$, but possibly different strings $)$. Then we have $\mu(x, w)=\mu\left(x^{*}, w^{*}\right)$.

(iv) If there exists $s \in \mathcal{L}(w) \backslash \mathcal{L}(x)$ then we have either $(a) \mu(x, w)=0$ or $(b)$ both $x=s w$ and $\mu(x, w)=1$.

(v) If there exists $s \in \mathcal{R}(w) \backslash \mathcal{R}(x)$ then we have either $(a) \mu(x, w)=0$ or $(b)$ both $x=w$ s and $\mu(x, w)=1$.

Definition 3.2 Suppose that $n$ is even. Let

$$
S_{0}=\left\{s_{0}, s_{2}, s_{4}, \ldots, s_{n-2}\right\}
$$

and

$$
S_{1}=\left\{s_{1}, s_{3}, s_{5}, \ldots, s_{n-1}\right\} .
$$

We define $I_{0}$ (respectively, $\left.I_{1}\right)$ to be the product in $W\left(\widetilde{A}_{n-1}\right)$ of the generators in $S_{0}$ (respectively, $S_{1}$ ). (Note that each of $I_{0}$ and $I_{1}$ is a product of $n / 2$ commuting generators.)

Proposition 3.3 (Shi) Let $w \in W\left(\widetilde{A}_{n-1}\right)$. If $w \notin W_{c}$, then there is a finite sequence $w=w_{0}, w_{1}, \ldots, w_{k}$ such that

(i) for each $0 \leq i<k$, we have $w_{i+1}={ }^{*} w_{i}$ with respect to some pair of noncommuting generators $\{s(i), t(i)\}$ depending on $i$, and 
(ii) $\mathcal{L}\left(w_{k}\right)$ is not commutative.

Proof This is a special case of [13, Lemma 2.2].

Proposition 3.4 (Fan-Green) Let $w \in W\left(\widetilde{A}_{n-1}\right)$. Suppose that $w \in W_{c}$. Then one of the following four situations must occur:

(i) $w$ is a product of commuting generators;

(ii) $n$ is even and $w$ is equal to an alternating product of the elements $I_{0}$ and $I_{1}$ in Definition 3.2;

(iii) we have $w=$ st $v$ reduced for a pair of noncommuting generators $I=\{s, t\}$, and so we have $t v={ }^{*} w$ with respect to $I$, for some $v \in W_{c}$;

(iv) we have $w=v t$ s reduced for a pair of noncommuting generators $I=\{s, t\}$, and so we have $v t=w^{*}$ with respect to $I$, for some $v \in W_{c}$.

Proof This is a restatement of [1, Proposition 3.1.2].

Lemma 3.5 Let $w \in W\left(\widetilde{A}_{n-1}\right)$ be fully commutative.

(i) The sets $\mathcal{L}(w)$ and $\mathcal{R}(w)$ are commutative (in the sense of Sect. 2).

(ii) Suppose $n$ is even, that $\mathcal{L}(w) \in\left\{S_{0}, S_{1}\right\}$, and that $\mathcal{R}(w) \in\left\{S_{0}, S_{1}\right\}$. Then $w$ is equal to an alternating product of $I_{0}$ and $I_{1}$.

(iii) If ${ }^{*} w$ is defined with respect to some pair $\{s, t\}$ of noncommuting generators, then ${ }^{*} w$ is fully commutative; the same is true for $w^{*}$.

Proof If $s$ and $t$ are noncommuting generators in $\mathcal{L}(w)$, then it follows using basic properties of Coxeter groups that $w$ has a reduced expression beginning st s, which is incompatible with the hypothesis $w \in W_{c}$. A similar argument deals with the case of $\mathcal{R}(w)$, and assertion (i) (which is well known) follows.

Assume the hypotheses of (ii). Suppose in addition that $\mathcal{L}(w)=S_{0}$, and assume (for a contradiction) that we are in (iii) of Proposition 3.4. In this case, we have $s \in S_{0}$. There will be precisely two generators that do not commute with $t$, both of which lie in $S_{0}$; let us call the other such generator $u$. The hypothesis $\mathcal{L}(w)=S_{0}$ shows that $u \in \mathcal{L}(w)$. Now $w=s t v$, being fully commutative, has the property that the occurrence of $t$ shown lies to the left of any occurrence of $u$, and this property is retained after repeated commutation of adjacent generators. It follows that $w$ has no reduced expression beginning in $u$, which is incompatible with $u \in \mathcal{L}(w)$ by the Exchange Condition for Coxeter groups. This is a contradiction.

Using similar arguments, we see that the case $\mathcal{L}(w)=S_{1}$ is incompatible with Proposition 3.4(iii), and the cases $\mathcal{R}(w)=S_{0}$ and $\mathcal{R}(w)=S_{1}$ are incompatible with Proposition 3.4(iv). Because of the assumption about $\mathcal{L}(w)$, the situation of Proposition 3.4(i) can only occur if $w=I_{0}$ or $w=I_{1}$, and these are special cases of Proposition 3.4(ii). The result follows.

Part (iii) is proved in [12, Proposition 2.10].

\section{Proof of main result}

Our main result is the following 
Theorem 4.1 Let $W$ be a Coxeter group of type $\widetilde{A}_{n-1}$, let $w \in W$ be arbitrary, and let $x \in W_{c}$. Then $\mu(x, w) \in\{0,1\}$.

Proof Suppose first that $w$ is not fully commutative.

If $\mathcal{L}(w)$ is not commutative, then Lemma 3.5(i) shows that we must have $s \in$ $\mathcal{L}(w) \backslash \mathcal{L}(x)$ and the result follows from Proposition 3.1(iv). Similarly, if $\mathcal{R}(w)$ is not commutative, then the result follows from Lemma 3.5(i) and Proposition 3.1(v).

Now let $w=w_{0}, w_{1}, \ldots, w_{k}$ be the sequence given in Proposition 3.3. We proceed by induction on $k$; the previous paragraph deals with the case $k=0$. Let $I=\{s, t\}$ be a pair of noncommuting generators with the property that $w_{1}={ }^{*} w_{0}$ with respect to $I$. We may assume without loss of generality that $s \in \mathcal{L}(w)$. If $s \notin \mathcal{L}(x)$, then we are done by Proposition 3.1(iv), so we may assume that $s \in \mathcal{L}(x)$. By Lemma 3.5(i), we have $t \notin \mathcal{L}(x)$. Since $\mathcal{L}(x) \cap I$ consists of a single element, we can apply a left star operation to $x$ with respect to $I$. By Proposition 3.1(ii), we have $\mu(x, w)=$ $\mu\left({ }^{*} x,{ }^{*} w\right)$. By Lemma 3.5(iii), ${ }^{*} x$ is fully commutative but ${ }^{*} w$ is not. We now repeat the argument with ${ }^{*} w$ in place of $w$, and the result follows by induction.

We may now suppose that $w \in W_{c}$. Proposition 3.4 shows that there are four cases to consider.

The first case is that $w$ is a product of commuting generators. In order for $\mu(x, w)$ not to be zero, we need $x<w$, which means that $x$ is a product of a proper subset of the aforementioned commuting generators, and that there exists $s \in \mathcal{L}(w) \backslash \mathcal{L}(x)$. The result then follows from Proposition 3.1(iv).

The second case is that $n$ is even and $w$ is an alternating product of $I_{0}$ and $I_{1}$. We may assume that $\mathcal{L}(w) \subseteq \mathcal{L}(x)$ and $\mathcal{R}(w) \subseteq \mathcal{R}(x)$, or we are done by Proposition 3.1(iv) and (v). Since $\mathcal{L}(x)$ and $\mathcal{R}(x)$ are commutative, we must have $\mathcal{L}(w)=$ $\mathcal{L}(x)$ (because $\left.\mathcal{L}(w) \in\left\{S_{0}, S_{1}\right\}\right)$ and $\mathcal{R}(w)=\mathcal{R}(x)$ (because $\left.\mathcal{R}(w) \in\left\{S_{0}, S_{1}\right\}\right)$. By Lemma 3.5(ii), $w$ and $x$ must both be alternating products of $I_{0}$ and $I_{1}$, and furthermore, both these alternating products must (a) start with the same $I_{i}$, and (b) end with the same $I_{j}$. It follows that $\ell(w)=\ell(x) \bmod 2$, which proves that $\mu(x, w)=0$ by Proposition 2.1 (i), and the result follows.

The third case for the element $w$ is the situation of Proposition 3.4(iii). We may assume that $s \in \mathcal{L}(x)$, or we are done by Proposition 3.1(iv). We also have $t \notin \mathcal{L}(x)$ by Lemma 3.5(i). Since $\mathcal{L}(x) \cap I$ is a singleton, the element ${ }^{*} x$ with respect to $I$ is defined. By Proposition 3.1(ii), we have $\mu(x, w)=\mu\left({ }^{*} x,{ }^{*} w\right)$, and by Lemma 3.5(iii), ${ }^{*} x$ and ${ }^{*} w$ are both fully commutative. By the assumptions on Proposition 3.4(iii), $\ell\left(^{*} w\right)=\ell(w)-1$, and the proof is completed by induction on $\ell(w)$.

The fourth case for the element $w$ is the situation of Proposition 3.4(iv), and we argue as in the previous paragraph, using Proposition 3.1(iii) and (v).

Remark 4.2 It follows easily from Proposition 3.1(i), (iv), (v) that Theorem 4.1 is also true in the case $n=2$.

It is possible to have $\mu(x, w)=1$ with $x$ and $w$ both fully commutative and $\ell(w)-\ell(x)>1$, for example, if $n \geq 4, x=s_{2}$ and $w=s_{2} s_{1} s_{3} s_{2}$.

Acknowledgements I thank Brant Jones for some helpful conversations and correspondence. I would also like to thank both referees for their constructive criticism and for reading the paper carefully. 


\section{References}

1. Fan, C.K., Green, R.M.: On the affine Temperley-Lieb algebras. J. L.M.S. 60, 366-380 (1999)

2. Humphreys, J.E.: Reflection Groups and Coxeter Groups. Cambridge University Press, Cambridge (1990)

3. Jones, B.C.: Leading coefficients of Kazhdan-Lusztig polynomials for Deodhar elements. J. Algebr. Comb. (in press). arXiv:0711.1391

4. Kazhdan, D., Lusztig, G.: Representations of Coxeter groups and Hecke algebras. Invent. Math. 53, 165-184 (1979)

5. Kazhdan, D., Lusztig, G.: Schubert varieties and Poincaré duality. Proc. Symp. Pure Math. 36, 185203 (1980)

6. Lascoux, A.: Polynômes de Kazhdan-Lusztig pour les variétés de Schubert vexillaires. C. R. Acad. Sci. Paris 321, 667-670 (1995)

7. Lascoux, A., Schützenberger, M.-P.: Polynômes de Kazhdan \& Lusztig pour les grassmanniennes. In: Young Tableaux and Schur Functors in Algebra and Geometry, Toruń, 1980. Asterisque, vol. 87-88, pp. 249-266. Soc. Math. France, Paris (1981)

8. Lusztig, G.: Some problems in the representation theory of finite Chevalley groups. Proc. Symp. Pure Math. 37, 313-317 (1980)

9. Lusztig, G.: Some examples of square integrable representations of semisimple $p$-adic groups. Trans. Am. Math. Soc. 277, 623-653 (1983)

10. Lusztig, G.: Cells in affine Weyl groups. In: Algebraic Groups and Related Topics. Adv. Studies Pure Math., vol. 6, pp. 255-287. North-Holland and Kinokuniya, Tokyo and Amsterdam (1985)

11. McLarnan, T.J., Warrington, G.S.: Counterexamples to the $0-1$ conjecture. Represent. Theory 7, 181195 (2003)

12. Shi, J.Y.: Fully commutative elements in the Weyl and affine Weyl groups. J. Algebra 284, 13-36 (2005)

13. Shi, J.Y.: Fully commutative elements and Kazhdan-Lusztig cells in the finite and affine Coxeter groups, II. Proc. Am. Math. Soc. 133, 2525-2531 (2005)

14. Stembridge, J.R.: On the fully commutative elements of Coxeter groups. J. Algebr. Comb. 5, 353-385 (1996)

15. Xi, N.: The leading coefficient of certain Kazhdan-Lusztig polynomials of the permutation group $S_{n}$. J. Algebra 285, 136-145 (2005) 